Neutral Orbital Altitude Density Effects on the International Space Station

\author{
O.E. Smith ${ }^{\dagger}$, S.I. Adelfang ${ }^{* \dagger}$ and R.E. Smith*
}

Computer Sciences Corporation, Huntsville, Alabama, 35824-6405

\begin{abstract}
One of the design requirements of the International Space Station (ISS) is that each year accelerations of one micro-g cannot be exceeded at the ISS internal payload location for 6 periods of not less than 30 consecutive days. Although there are other causes, this study deals only with the accelerations caused by atmospheric drag. The critical ambient neutral density, computed using the Marshall Engineering Thermosphere Model, required to produce accelerations of one micro-g on the ISS, is estimated using an atmospheric drag acceleration equation. Results show that the design requirements may be difficult to meet during periods of extremely high solar activity; the planned reboost and altitude strategies for the ISS may have to be revised to allow for the uncertainty in the prediction of neutral atmospheric density within the 100-day period established for orbital decay before reboost
\end{abstract}




\title{
Neutral Orbital Altitude Density Effects on the International Space Station \\ O.E. Smith* ${ }^{\dagger}$, S.I. Adelfang ${ }^{{ }^{\dagger}}$ and R.E. Smith* \\ Computer Sciences Corporation, Huntsville, Alabama, 35824-6405
}

\begin{abstract}
One of the design requirements of the International Space Station (ISS) is that each year accelerations of one micro-g cannot be exceeded at the ISS internal payload location for 6 periods of not less than 30 consecutive days. Although there are other causes, this study deals only with the accelerations caused by atmospheric drag. The critical ambient neutral density, computed using the Marshall Engineering Thermosphere Model, required to produce accelerations of one micro-g on the ISS, is estimated using an atmospheric drag acceleration equation. Results show that the design requirements may be difficult to meet during periods of extremely high solar activity; the planned reboost and altitude strategies for the ISS may have to be revised to allow for the uncertainty in the prediction of neutral atmospheric density within the 100-day period established for orbital decay before reboost
\end{abstract}

\section{NOMENCLATURE}

$F_{10.7}$ daily value of $10.7 \mathrm{~cm}$ solar radio noise flux, units are $10^{-22}$ watts $/ \mathrm{m}^{2} / \mathrm{Hz}$ bandwidth or $10^{4}$ Jansky

$\bar{F}_{10.7}$ average value of $F_{10.7}$

i inclination, $51.6 \mathrm{deg}$

K degrees Kelvin

$r_{0} \quad$ Earth's equatorial radius, $6,378,140 \mathrm{~m}$

*Senior Scientist, Applied Technology, PrISMS, P.O. Box 240005, Huntsville, AL 35824. $\uparrow$ Member AIAA 
$V_{c} \quad$ satellite circular velocity, $\mathrm{m} / \mathrm{s}$

Z altitude above the Earth, $m$

$\mu \quad$ Earth's gravitational parameter, $3.986012 \times 10^{14} \mathrm{~m}^{3} \mathrm{~s}^{-2}$

$\rho \quad$ neutral density, $\mathrm{kg} / \mathrm{m}^{3}$

$\rho_{Z} \quad$ density at altitude $Z$

$\omega \quad$ Earth's rotation rate $\left(7.2921 \times 10^{-5} \mathrm{rad} / \mathrm{s}\right)$

\section{INTRODUCTION}

Both the micro-gravity experiment acceleration requirements and the altitude and reboost strategies of the ISS are dependent on the combination of the ballistic coefficient of the ISS and the neutral orbital altitude mass density. Predicting the orbital altitude neutral mass density for the time period of the assembly and operation of the International Space Station (ISS) with any accuracy is extremely difficult because the exact relationship between the solar inputs that affect the atmosphere and the magnitude of the change they cause are poorly known. To make matters worse predictions of the proxy solar inputs used in the Marshall Engineering Thermosphere (MET) model are even more difficult.

This study estimates what might occur in the future by simulating ISS operations during selected periods in the historical data base of required inputs to the neutral atmosphere model used in the ISS development program. Results are compared to the ISS design requirements and planned reboost and altitude strategies. The data base of inputs required for the MET model of the neutral thermosphere extends from 1947 through 1994.

\section{BACKGROUND}


NASA has specified that the MET model of the neutral atmosphere is to be used in the design, development, and testing phases of the ISS ${ }^{1}$. The MET model is a computer code ${ }^{2,3}$ based on the Jacchia $70^{4}$ and the Jacchia $71^{5}$ (for seasonal variation) thermosphere models. The Jacchia $70^{4}$ atmospheric model documentation states that the density output of the model was referenced to the 81-day mean values of the $10.7 \mathrm{~cm}$ solar radio noise flux, $\overline{\mathrm{F}}_{10.7}$. Discussions and studies conducted in the middle to late 1970 s and a recent study ${ }^{6}$ established that use of the 81-day running mean prior to the time of application produced predicted lifetimes for Earth orbiting satellites that were in better agreement with the actual lifetimes than the use of 162-day average $10.7-\mathrm{cm}$ values required for implementation of the original version of the MET model ${ }^{2}$. Although the decay histories of satellites can be acceptably duplicated after the fact by using the MET model with the actual parameters designated in the original documentation, decay histories of satellites at future times depend entirely upon the accuracy of (1) the ballistic coefficient and (2) the proxy solar parameters required as inputs to the model. Results of studies that were conducted to determine what $F_{10.7}$ values could be predicted for use in the prediction of decay rates at future times showed that values of the $10.7 \mathrm{~cm}$ solar radio noise flux and the 3-hourly average geomagnetic index averaged over less than 13-months could not be predicted with sufficient accuracy for the application.

\section{DISCUSSION}

The neutral atmosphere parameter that affects the micro-gravity acceleration design requirement and the altitude strategy plan of the ISS is the ambient mass density. This analysis of these two portions of the ISS program uses the 1947-1994 historical database of the parameters that are 
required inputs to the MET model. It is in essence a look at the ISS as if it were in orbit at selected time periods between 1947 and 1994, primarily periods with high levels of solar activity.

Calculation of ambient mass density with the MET model is based on determination of the temperature structure above $90 \mathrm{~km}$ altitude, which is determined by construction of a temperature profile that begins at $90 \mathrm{~km}$ with a fixed boundary condition (defined below) and ends àt about $350 \mathrm{~km}$ where the temperature is defined as the exospheric temperature. The exospheric temperature is the key variable for determination of the variability of ambient orbital density. The exospheric temperature is highly dependent on the extreme ultraviolet (EUV) output of the Sun and the solar storms which inject energetic particles into the Earth's atmosphere. Since neither of these parameters are measurable at the Earth's surface, proxy parameters that are available are used as inputs to the model. The $10.7-\mathrm{cm}$ solar radio noise flux, both the average and the daily value, is a proxy for the EUV while the 3-hourly average geomagnetic index, $a_{p}$, is a proxy for the Joule heating of the atmosphere that results from the deposition of the energetic particles.

The MET model is used to compute total mass density from 250 to $500 \mathrm{~km}$ altitude using the available 47 years (1947-1994) daily values for the $10.7-\mathrm{cm}$ solar flux and the 3 -hourly ( 8 per day) average values of the geomagnetic index, $a_{p}$. These mass densities are then used (1) to compute micro-gravity levels as a function of altitude for comparison with the specified design requirements and (2) in orbital decay analyses for comparison with the altitude strategy plan.

Once the variations in the exospheric temperature over the globe are computed, the only other parameter needed to describe the thermosphere density is the fixed boundary condition at $90 \mathrm{~km}$ altitude, which is a density value of $3.46 \times 10^{-6} \mathrm{~kg} \mathrm{~m}^{-3}$, an ambient temperature of $183 \mathrm{~K}$ and a mean molecular mass $\overline{\mathrm{M}}_{90}=28.878 \mathrm{~g} \mathrm{~mole}^{-1}$. 
The appendix, Table A, gives mass density versus altitude $[250(\delta 10) 500 \mathrm{~km}]$ and exospheric temperature $[600(\delta 200) 2200 \mathrm{~K}]$. This convenient reference table for density was computed using the MET model for the assigned values of $T_{E}$. Table A covers the planned operating altitude range for the ISS. The values for density match those from the Jacchia 70 model $^{4}$ for $T_{E}$ greater than $700 \mathrm{~K}$; at $600 \mathrm{~K}$ the differences in density are less than one percent at $500 \mathrm{~km}$ altitude. This density difference is attributed to the difference in the numerical integration techniques used to evaluate the diffusion equation. The density from Table $\mathrm{A}$ is illustrated in Fig. 1.

(Fig. 1)

Density variations in the MET model are based on:

1. The $10.7 \mathrm{~cm}$ solar radio noise flux which was measured at Ottawa, Canada from 1947 to June 1991, and since June 1991 is measured at Penticton BC, Canada.

2. The planetary geomagnetic index. This index is made available through the International Union of Geophysics and Geodesy (IUGG), Gottingen, Germany.

3. The rotation of the Earth (diurnal variation).

4. The Earth's rotation about the Sun (semi-annual variation).

5. Seasonal-latitudinal variations below $170 \mathrm{~km}$ altitude. This variation is established by a set of empirical equations that correct the mass density between 90 and $170 \mathrm{~km}$ altitude. This variation does not affect density above $170 \mathrm{~km}$.

6. Seasonal-latitudinal variations in helium for altitudes greater than $500 \mathrm{~km}$.

The required input parameters for this application of the MET model are:

1. Date (month, day, year),

2. Greenwich mean time (GMT) from midnight, $0000 \mathrm{GMT}$, in hours and minutes, 
3. Latitude (deg),

4. Longitude (deg),

5. Altitude (km)

6. The daily value for the $10.7-\mathrm{cm}$ solar flux, $F_{10.7}$ for the day prior to the date of interest,

7. The 81-day average $10.7-\mathrm{cm}$ solar flux, $\vec{F}_{10.7},(81$ days prior to day of interest)

8. The 3-hourly planetary geomagnetic index, $a_{p}, 6.7$ hours prior to the time of interest.

The key equations in the MET model that relate to the solar flux and the planetary geomagnetic index are presented below. The nighttime minimum global exospheric temperature, $T_{C}$, when the geomagnetic index, $a_{p}$, is zero, is:

$$
\mathrm{T}_{\mathrm{C}}=383+3.32 \overline{\mathrm{F}}_{10.7}+1.8\left(\mathrm{~F}_{10.7}-\overline{\mathrm{F}}_{10.7}\right)
$$

where, $F_{10.7}$ is the daily value and $\bar{F}_{10.7}$ is the average value of the solar flux. In this equation the empirical coefficients ensure correct units.

The daytime maximum exospheric temperature, $T_{D}$, is:

$$
\mathrm{T}_{\mathrm{D}}=\mathrm{RT}_{\mathrm{C}}
$$

where, $R$, a function of the 400 day average solar flux, varies from 0.27 to 0.40 and has an average value of $0.31^{4}$.

The variation in the exospheric temperature due to the 3-hourly geomagnetic index, $a_{p}$, is:

$$
\Delta \mathrm{Tg}=1.0 \mathrm{a}_{\mathrm{p}}+100\left[1-\exp \left(-0.08 \mathrm{a}_{\mathrm{p}}\right)\right]
$$

The $\mathrm{a}_{\mathrm{p}}$ index has only discrete values between zero and 400 while $\Delta \mathrm{Tg}$ ranges from 0 to $500 \mathrm{~K}$. The variable, $\Delta \mathrm{Tg}$, is the single most important contributor to exospheric temperature change within a day. Three hours is the highest time resolution obtainable from the MET model due to the 3-hour intervals for $a_{p}$. 
The semi-annual contribution to the exospheric temperature $(\mathrm{K})$ variation is:

$$
\Delta \mathrm{Ts}=2.41+\overline{\mathrm{F}}_{10.7} \mathrm{~g}(\mathrm{t})
$$

where,

$$
g(t)=\left[0.349+0.206 \sin \left(360^{\circ} \tau+226.5^{\circ}\right)\right]\left[\sin \left(720^{\circ} \tau+247.6^{\circ}\right]\right.
$$

and

$$
\tau=\frac{\mathrm{d}}{\mathrm{y}}+.1145\left[\left(\frac{1+\sin \left(360^{\circ}(\mathrm{d} / \mathrm{y})+342.3^{\circ}\right.}{2}\right)^{2.16}-1 / 2\right]
$$

where, $d$ is days since January 1 and $y$ is the length of the tropical year, 365.2422 days. Fig. 2 presents the evaluations for $g(t)$.

(Fig. 2)

The global maximum exospheric temperature is:

$$
\mathrm{Tmax}=(1.0+\mathrm{R}) \mathrm{T}_{\mathrm{C}}+\Delta \mathrm{Tg}+\Delta \mathrm{Ts} .
$$

This value occurs at the latitude of the subsolar point with a time lag of approximately 2 hours or, at 1400 hours local Sun time. At any altitude in the thermosphere the largest density occurs at the location of Tmax.

The minimum exospheric temperature is:

$$
\mathrm{Tmin}=\mathrm{T}_{\mathrm{C}}+\Delta \mathrm{Tg}+\Delta \mathrm{Ts} .
$$

The global averages for density and exospheric temperature in simplified satellite orbital decay models are estimated from:

$$
\begin{array}{r}
\bar{\rho}_{G}=\frac{1}{2}\left[\rho\left(T_{\text {max }}\right)+\rho\left(T_{\text {min }}\right)\right] \\
\text { Tavg }=(1+R / 2) T_{C}+\Delta T s+\Delta T g
\end{array}
$$


For applications requiring determination of the density along a satellite trajectory in orbit the exospheric temperature as a function of latitude, longitude, and time is required. This local exospheric temperature is:

$$
T_{L}=T_{C}\left(1+R \sin ^{m} \theta\right)\left[1+R\left(\frac{\cos ^{m} \eta-\sin ^{m} \theta}{1-R \sin ^{m} \theta}\right) \cos ^{n} \tau / 2\right]
$$

where, $\tau=H+\beta+p \sin (H+\gamma),(-\pi<\tau<\pi), H$ is the hour angle of the Sun, $m=2.5, n=3.0$, $\beta=-37 \mathrm{deg}, p=+6 \mathrm{deg}, \gamma=+43 \mathrm{deg}$, and $\eta=1 / 2|\mathrm{LAT}-\mathrm{DL}|, \theta=1 / 2|\mathrm{LAT}+\mathrm{DL}|$, where, LAT is the latitude of the satellite and DL is the Sun declination angle.

The local exospheric temperature, $T_{E}$, is then:

$$
\mathrm{T}_{\mathrm{E}}=\mathrm{T}_{\mathrm{L}}+\Delta \mathrm{Tg}+\Delta \mathrm{Ts}
$$

There are sets of empirical equations that define the temperature versus altitude, $T_{2}$, between the $T$ at $90 \mathrm{~km}\left(183 \mathrm{~K}\right.$ ) and $\mathrm{T}_{\mathrm{E}}$ (at about $350 \mathrm{~km}$ ) that are functions of altitude and the exospheric temperature only. The hydrostatic equation is numerically integrated using these functions, $T_{Z}$, and the boundary conditions at $90 \mathrm{~km}$ altitude to obtain the neutral mass density up to $105 \mathrm{~km}$ altitude. For altitudes above $105 \mathrm{~km}$ the diffusion equation is used to compute number densities of the atmospheric constituents and total mass density. For altitudes above $500 \mathrm{~km}$ a correction is made to the density for the seasonal variation in helium.

The value for $T_{E}$ (Eq. 9) lies between $T \min (E q .6)$ and $T \max (E q . ~ 5)$, i.e.,

$$
\mathrm{T} \min \leq \mathrm{T}_{\mathrm{E}} \leq \mathrm{T} \max
$$

The orbit of a low earth orbiting satellite will retrograde approximately 4 degrees per day; considering this, there is a good chance that any satellite with an inclination $\geq 23.45$ degrees will pass through Tmin and Tmax at least one time in 30 days. 


\section{ANALYSIS}

Fig. 3 presents the daily values for the $10.7 \mathrm{~cm}$ solar flux for the period of record under study. This period covers the latter part of solar cycle 18, all of cycles 19, 20, 21 and most of cycle 22 . The 3-hourly planetary geomagnetic index, $a_{p}$, for this period of record is shown in Fig. 4 . The daily and 81-day prior average (in this case) solar flux and $a_{p}$ are used to compute the daytime daily maximum global exospheric temperature shown in Fig. 5 (top curve); the differences between the daily global maximum (Eq. 5) and daily global minimum (Eq. 6) exospheric temperature are also shown in Fig. 5 (bottom curve).

(Figures 3, 4 and 5)

The largest daily maximum exospheric temperature, $2152.2 \mathrm{~K}$ and the largest thermospheric density, as computed by the MET model, occurred during solar cycle 19 on September 23, 1957 at $0600 \mathrm{GMT}$. The density values versus altitude can be read from Table $\mathrm{A}$ for $\mathrm{T}_{\mathrm{E}}=2150 \mathrm{~K}$. For example, at $450 \mathrm{~km}, 400 \mathrm{~km}$ and $300 \mathrm{~km}$, the density values are: $0.15699 \times 10^{-10}, 0.26025 \times 10^{-10}$ and $0.83120 \times 10^{-10}$, respectively. Table $\mathrm{A}$ can be used to obtain the density for any arbitrarily assigned or known value for the exospheric temperature.

The ISS Microgravity Experiment Requirement from the System Specification Document for the $\operatorname{ISS}^{1}$ is:

"The Space Station shall provide the following microgravity acceleration performance for at least 50 percent of the internal payload locations for 180 days per year in continuous time intervals of at least 30 days. At the centers of the internal payloads, a quasi-steady $(<0.01 \mathrm{~Hz})$ acceleration: (a) magnitude less than or equal to one micro-g and (b) 
component perpendicular to the orbital average acceleration vector less than or equal to 0.2 micro-g."

There are also vibration acceleration limits for the frequency range, $0.01 \leq \mathrm{f} \leq 300 \mathrm{~Hz}$.

The variability of atmospheric density during one micro-gravity experiment (30 days duration) and the ISS response during this period are important factors that contribute to the residual acceleration.

\section{Atmospheric Drag Acceleration}

The atmospheric drag acceleration, $D,\left(\mathrm{~ms}^{-2}\right)$ is given by:

$$
\mathrm{D}=\frac{1}{2} \mathrm{C}_{\mathrm{D}}\left(\frac{\mathrm{A}}{\mathrm{m}}\right) \mathrm{v}_{\mathrm{rel}}^{2} \rho
$$

where $C_{D}$ is $2.2, A$ is $2673 \mathrm{~m}^{2}$, and $\mathrm{m}$ is $420,000 \mathrm{~kg}$ for the ISS. These estimates yield:

$$
\mathrm{C}_{\mathrm{D}} \frac{\mathrm{A}}{\mathrm{m}}=0.014\left(\mathrm{~m}^{2} / \mathrm{kg}\right)
$$

$\rho$ is atmospheric density and $V_{\text {rel }}$ is the relative velocity between the ISS and the atmosphere. For a spherical Earth and a circular orbit $V_{\text {rel }}$ is a function of the circular velocity $\left(V_{C}\right)$ at altitude $(Z)$, inclination (51.6 deg for the ISS) and latitude. At zero deg latitude and an inclination of $51.6 \mathrm{deg}$, $V_{\text {rel }}$ at $Z=300 \mathrm{~km}$ is $7422 \mathrm{~m} / \mathrm{s}$ and at $Z=400 \mathrm{~km}, V_{\text {rel }}$ is $7359 \mathrm{~m} / \mathrm{s}$. For illustration purposes $V_{\text {rel }}$ is taken as $7400 \mathrm{~m} / \mathrm{s}$. From the above parameters and assumptions, the atmospheric drag acceleration $\mathrm{D}$ (Eq. 11) can be approximated by:

$$
\mathrm{D}=383320 \rho
$$

To convert Eq. 12 to micro-gs ( $\mu-\mathrm{g}$ ) divide by $\mathrm{g}_{0 \times} 10^{-6}$, where $\mathrm{g}_{0}$ is $9.80665 \mathrm{~ms}^{-2}$ :

$$
\mu-g=39089 \times 10^{6} \rho .
$$


For example, when $\rho=2.5583 \times 10^{-11}$, Eq. 13 gives one micro-g. Hence, when $\rho \geq 2.5583 \times$ $10^{-11}$, the atmospheric drag acceleration equals or exceeds one micro-g. Fig. 6 shows $\rho=2.5583 \mathrm{x}$ $10^{-11} \pm 20 \%$, which is a reasonable uncertainty for the MET model, as a function of exospheric temperature and altitude. The values in Fig. 6 are important in planning altitude strategies for the micro-gravity experiments. The curves in Fig. 6 represent the exospheric temperature to be used to estimate the altitude above which one-micro-g will not be exceeded if the MET model density : (1) has no uncertainty (curve A); (2) is underestimated by 20 percent (curve B); (3) is overestimated by 20 percent (curve C). To protect the ISS for the worst case, which is when MET underestimates the density, curve B is applicable. For no uncertainty in the density (curve A) one micro-g acceleration would not have been exceeded at altitudes greater than $397 \mathrm{~km}$ because $T_{E}$ is not greater than $2152.2 \mathrm{~K}$ (Fig. 5) for the entire period of record (1947-1994). To protect for the worst case uncertainty the altitude would have to be at least $422 \mathrm{~km}$ to ensure that one micro-g is not exceeded for the entire period of record (curve B). Similarly, if protection is required for solar activity levels associated with $T_{E} \leq 1700 \mathrm{~K}$, which excludes very high solar activity, the minimum altitude is $363 \mathrm{~km}$ for no uncertainty (curve A) and $380 \mathrm{~km}$ for the worst case (curve B).

(Fig. 6)

A comparison of the mass spectrometer measurements from the Atmospheric Explorer-E (AEE) satellite with the density computed from the MET model using the 162-day centered average 10.7-cm solar flux, the 81-day prior average solar flux and the $1 / 2$ gaussian weighted prior average solar flux revealed that a better agreement exists between the MET model and the AE-E densities using the 81-day average flux or the 81-day gaussian average flux than the 162-day 
average solar flux ${ }^{6}$. The gaussian average flux, the daily flux and $a_{p}$ shown in Fig. 7 for July 1959 were used in the MET model for the analysis of the ISS in circular orbit at $300 \mathrm{~km}$ altitude (Fig. 8). As shown the density exceeded the critical value for one-micro-g for most of the orbits for the entire month of July 1959. This example is for high solar activity. For periods of low solar activity one-micro-g is not exceeded for 30 or more days. For this paper $T_{E}>1500 \mathrm{~K}$ is considered high solar activity, $\mathrm{T}_{\mathrm{E}}<1000 \mathrm{~K}$ is low solar activity and the values between 1000 and $1500 \mathrm{~K}$ are intermediate solar activity.

(Figures 7 and 8)

\section{The Variability of Density within An Orbit}

The variability of atmospheric density within an orbit is required for use in ISS engineering analyses of (1) the control moment gyros (CMGs') capability to control the torques produced by the difference between the center of pressure and the center of gravity; and (2) the atmospheric drag acceleration that may exceed one-micro-g. Fig. 9 for July 15-16, 1959 shows that the variation in density within an ISS orbit at $300 \mathrm{~km}$ altitude can exceed $2.6 \times 10^{-11} \mathrm{~kg} \mathrm{~m}^{-3}$ or onemicro-g.

(Fig. 9)

To obtain the upper limits on the range of density for this purpose, the largest 3-hour change in the exospheric temperature from the MET model was computed. The exospheric temperatures associated with the largest daily 3-hour changes $\left(T_{1}, T_{2}\right)$ are shown in Fig. 10; the range of temperature for $T_{E}>1000 \mathrm{~K}$ is approximately $\pm 200 \mathrm{~K}$. Densities for $T_{E}=1600 \mathrm{~K} \pm 200 \mathrm{~K}$ at 400 and $300 \mathrm{~km}$ from Table A are listed in Table 1.

(Fig. 10) 
Table 1. Maximum 3-hour change in thermosphere density for $T_{E}=1600 \pm 200 \mathrm{~K}$

\begin{tabular}{lcccccc}
\hline $\mathrm{T}_{\mathrm{E}}(\mathrm{K})$ & $\begin{array}{c}\rho \\
\mathrm{kg} \mathrm{m}^{-3}\end{array}$ & $\begin{array}{c}400 \mathrm{~km} \\
\mathrm{~kg} \mathrm{~m}^{-3}\end{array}$ & $\begin{array}{c}\Delta \rho \text { rel } \\
\text { percent }\end{array}$ & $\begin{array}{c}\rho \\
\mathrm{kg} \mathrm{m}^{-3}\end{array}$ & $\begin{array}{c}300 \mathrm{~km} \\
\Delta \rho \\
\mathrm{kg} \mathrm{m}^{-3}\end{array}$ & $\begin{array}{c}\Delta \rho \text { rel } \\
\text { percent }\end{array}$ \\
\hline 1400 & $1.0136 \times 10^{-11}$ & $-0.4118 \times 10^{-11}$ & -29 & $2.2861 \times 10^{-11}$ & $-2.5213 \times 10^{-11}$ & -52 \\
1600 & $1.4324 \times 10^{-11}$ & 0.0 & 0 & $4.8074 \times 10^{-11}$ & 0.0 & 0 \\
1800 & $1.8642 \times 10^{-11}$ & $0.4318 \times 10^{-11}$ & 30 & $6.9264 \times 10^{-11}$ & $2.1191 \times 10^{-11}$ & 44 \\
& & & & & & \\
\hline \hline
\end{tabular}

\section{ISS Orbital Decay}

Three different models are used to compute the ISS orbital decay. They are: (Model 1) A complete orbital decay model whereby the decay rate is calculated for each orbit by integrating the densities (computed using the MET model with the prior 81-day means, prior day value and the value of $a_{p} 6.7$-hours prior to the time of interest) at 90 evenly-spaced points around the orbit; (Model 2) A simple orbital decay model (described later) using a single average global thermosphere density computed from Eq. 7 based on the same inputs to the MET model as Model 1 and updated at 3-hour intervals; (Model 3) same as (Model 2) but using the 13-month smoothed $10.7 \mathrm{~cm}$ solar flux and 13 -month smoothed geomagnetic index, $a_{p}$, in the MET model with the daily flux set equal to the 13-month smoothed solar flux. The results for the complete orbital decay model for ISS initial altitudes of 450 and $400 \mathrm{~km}$ beginning January 22, 1958, a high solar activity condition, are illustrated in Fig. 11.

(Fig. 11)

A simple orbital decay model for a circular orbit can be expressed as:

$$
\frac{d Z}{d t}=C_{D}\left(\frac{A}{m}\right)\left(r_{o}+Z\right) \rho_{Z} V_{c}\left[1-\left(\frac{\omega\left(r_{o}+Z\right) \cos i}{V_{c}}\right)^{2}\right]
$$


where $r_{0}=6,378,140 \mathrm{~m}, Z$ is altitude above the earth, $\rho_{Z}$ is the density at altitude $Z(m), V_{c}$ is the satellite circular velocity $(\mathrm{m} / \mathrm{s})$ where

$$
V_{c}=\left[\frac{\mu}{\left(r_{o}+Z\right)}\right]^{\frac{1}{2}},
$$

and $\mu=3.986012 \times 10^{14} \mathrm{~m}^{3} \mathrm{~s}^{-2}, \omega=7.2921 \times 10^{-5} \mathrm{rad} / \mathrm{s}, \mathrm{i}=51.6 \mathrm{deg}$. The other parameters are as defined in Eq. $11 ; \frac{\mathrm{dz}}{\mathrm{dt}}$ is the orbital decay rate $(\mathrm{m} / \mathrm{s})$.

From Model 1 ISS decayed from the initial altitude of $400 \mathrm{~km}$ to $253 \mathrm{~km}$ in 90 days. From Model 2 (Eq. 14), using the same solar inputs as in Model 1, the ISS altitude decayed from 400 to $263 \mathrm{~km}$ in 90 days. From Model 3 the ISS altitude decayed from 400 to $240 \mathrm{~km}$ in 90 days (lower curves, Fig. 11). For the ISS at an initial altitude of $450 \mathrm{~km}$ (upper 3 curves in Fig. 11) all three orbital decay models yield nearly coincident values of decay to $405 \mathrm{~km}$ in 90 days. This analysis suggests that the simple orbital decay model (Eq. 14 using the average global density) is a good approximation for the ISS orbital decay.

The 100-day ISS orbital decay from initial altitudes of $400 \mathrm{~km}$ and $450 \mathrm{~km}$ was computed using the simple orbital decay model (Eq. 14) with inputs to the MET model of the 81-day prior average solar flux, daily solar flux and $a_{p}$ at 6 hours prior to the time of computation to compute the global maximum and minimum exospheric temperature (Eqs. 5 and 6) and then the global average density was computed(Eq. 7). The results are shown in Fig. 12 (solid lines) for the years 1948 to 1994 . In a similar manner, the 13-month smoothed solar flux, and 13-month smoothed $a_{p}$ were used in the MET model to compute the global average density. The daily solar flux was set equal to the 13-month smoothed solar flux. These results for 100 days decay are shown as dots in Fig. 12. For initial altitudes of 400 and $450 \mathrm{~km}$ there is good agreement between the two MET 
model inputs(81-day prior and 13-month smoothed) to the simple orbital decay model. For solar cycle 19 and an initial altitude of $400 \mathrm{~km}$ the orbital decay in 100 days is so large that there is a risk indicated that the ISS will reenter during high solar activity unless reboosted. In contrast, the orbital decay during low solar activity for the initial altitude of $400 \mathrm{~km}$ is approximately $10 \mathrm{~km}$ in 100 days. The variation in orbital decay for various initial altitudes and durations is an important consideration in planning re-boost strategies for the ISS.

(Fig. 12)

The ISS assembly is to be completed in the year 2002. Assuming that solar cycle 23 began near mid-year 1996 places part of the ISS assembly period near the peak of the cycle when solar activity effects on orbital decay could be significant.

\section{CONCLUSIONS}

Based on the MET model for estimation of neutral mass density at ISS orbital altitudes for the entire period of record (1947-1994) for the model input variables that produced a calculated maximum value for exospheric temperature of $2152.2 \mathrm{~K}$, the altitude above which the atmospheric drag acceleration on the ISS would not have exceeded one micro-g is $397 \mathrm{~km}$ for no uncertainty in the MET model density and $422 \mathrm{~km}$ if the MET model underestimates the density by 20 percent (the worst case uncertainty). These altitudes are reduced to $363 \mathrm{~km}$ (no uncertainty) and $380 \mathrm{~km}$ (worst case 20 percent uncertainty) if the maximum exospheric temperature is less than $1700 \mathrm{~K}$. The range of this critical altitude versus exospheric temperature (Fig. 6) for one micro-g has important implications for the ISS planning strategies.

For the ISS orbital decay up to 100 days for initial altitudes at 400 and $450 \mathrm{~km}$, the use of the 13 month smoothed values for the $10.7 \mathrm{~cm}$ solar flux and the geomagnetic index $\left(a_{p}\right)$ produces 
results which are comparable to those produced by the using the specified inputs to the MET model. For periods of high solar activity, as in solar cycle 19 (Fig. 12), this study suggests the ISS could re-enter the earth's atmosphere within 100 days from an initial altitude of $400 \mathrm{~km}$ if not reboosted.

For development of re-boost operational strategies and for real time operations the complete MET model with 81-day running means and daily values for $10.7 \mathrm{~cm}$ solar flux and 3-hourly values for $a_{p}$ are required.

The variation in density within an ISS orbit is important information for the assessment of the capability of the ISS to meet the micro-g experiment acceleration requirements and requirements for torque and attitude control by the control moment gyros.

\section{ACKNOWLEDGMENTS}

This work was performed under a NASA George C. Marshall Space Flight Center contract to Computer Sciences Corporation (CSC PrISMS). The authors thank our sponsor, B. J.(Jeff) Anderson, MSFC Systems Analysis and Integration Laboratory, for helpful discussions in developing the scope of this effort. We thank C.G. Justus, the CSC team leader, for helpful discussions during the preparation of this paper and Ms. Belinda Hardin, administrative assistant, for preparing the manuscript.

The authors acknowledge with gratitude our colleague William J. Cooke (CSC) for his formulation of the orbital decay models used in this study.

The authors appreciate the comments and suggestions of the anonymous reviewer and the editor, Iain D. Boyd. 


\section{REFERENCES}

${ }^{1}$ Anon, "System Specification for the International Space Station Alpha," Specification Number 41000B, Cage Identification No. 2B945, NASA, 1 Nov. 1994.

${ }^{2}$ Hickey, M. P., "The NASA Marshall Engineering Thermosphere Model." NASA CR-179359, May, 1988.

${ }^{3}$ Hickey, M. P., "An Improvement in the Integration Procedure Used in the NASA Marshall Engineering Thermosphere Model." NASA CR-179389, August, 1988.

${ }^{4}$ Jacchia, L. G., "New Static Models of the Thermosphere and Exosphere with Empirical Temperature Profiles," Smithsonian Astrophysical Observatory, Report No. 313, May, 1970.

${ }^{5}$ Jacchia, L. G., "Revised Static Models of the Thermosphere and Exosphere with Empirical Temperature Profiles," Smithsonian Astrophysical Observatory, Report No. 332, May, 1971.

${ }^{6}$ Smith, O.E., S. I. Adelfang, R. E. Smith, C. G. Justus, and B. J. Anderson, "On-Orbit Ambient Mass Density for Space Station Operational Planning," AIAA 96-0631. Proceedings of the 34th Aerospace Sciences Meeting and Exhibit, January 15-18, 1996, Reno, NV. 


\section{Appendix \\ Table A. MET model neutral mass density $\left(\mathrm{kg} / \mathrm{m}^{3}\right)$ as a function of altitude and exospheric temperature.}

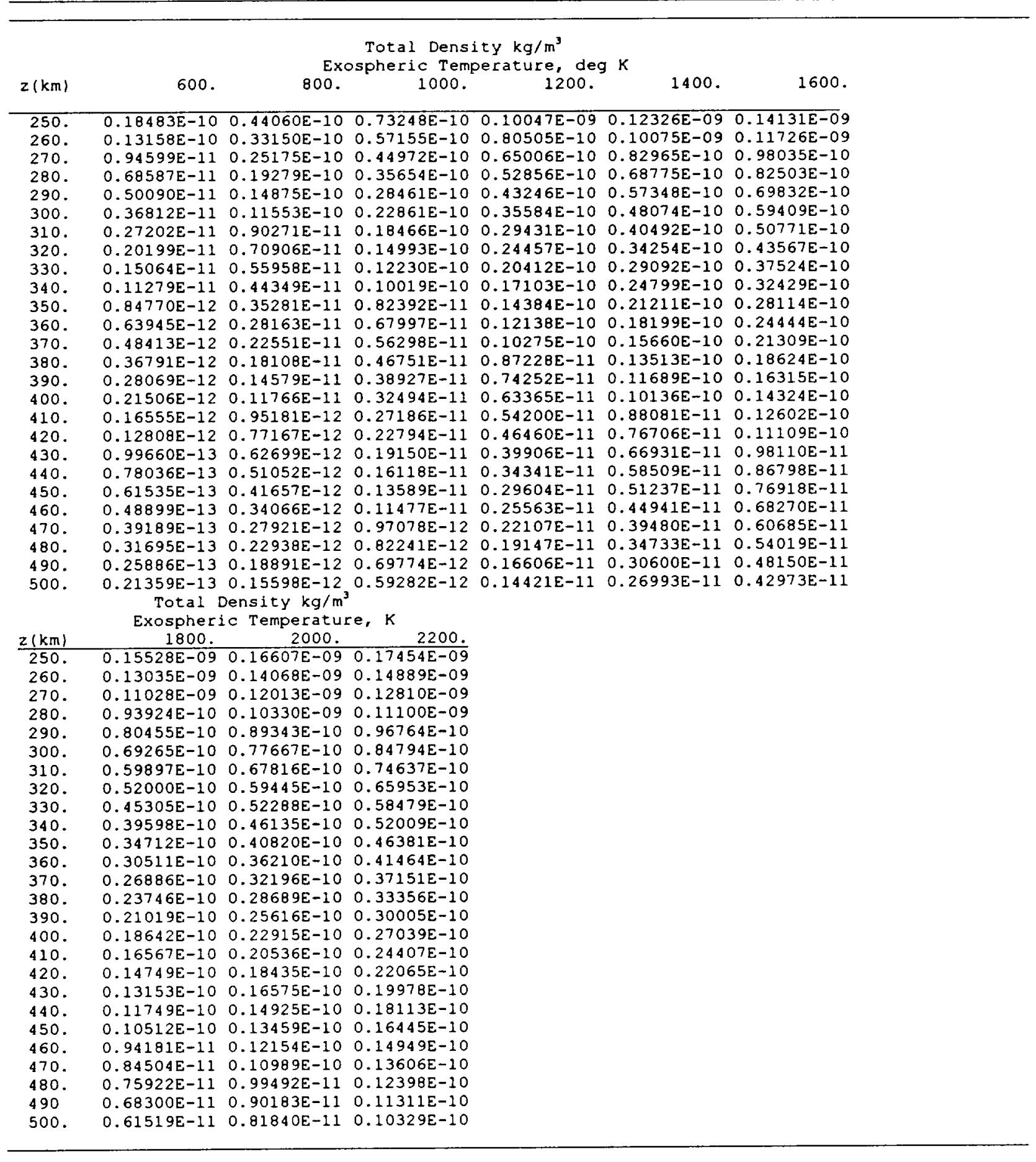


JSR Document Log.No. A10420

\section{$\underline{\text { List of Figures }}$}

Fig. 1 Density-altitude versus exospheric temperature from Table A.

Fig. 2 Term $g(t)$ for semi-annual variation in exospheric temperature.

Fig. 3 Daily values for $10.7-\mathrm{cm}$ solar flux for period of study.

Fig. 4 Planetary geomagnetic index (3-hourly $a_{p}$ )

Fig. 5 Daily maximum global exospheric temperature (top curve) and the differences in daily maximum and daily minimum global exospheric temperature (bottom curve).

Fig. 6 Altitude versus exospheric temperature for (A)

$\rho^{*}=2.5583 \times 10^{-11} \mathrm{~kg} / \mathrm{m}^{3}=1 \mathrm{micro}-\mathrm{g}$; (B) $.80 \rho^{*}$, and (C) $1.20 \rho^{*}$ for the ISS.

Fig. 7 Solar activity data for July 1959.

Fig. 8 MET model density using 81-day Gaussian mean $10.7 \mathrm{~cm}$ solar flux for ISS orbits at $300 \mathrm{~km}$ altitude for July 1959.

Fig. 9 MET model density using 81-day Gaussian mean $10.7 \mathrm{~cm}$ solar flux for ISS orbits at $300 \mathrm{~km}$ altitude for 15-16 July 1959.

Fig. 10 Exospheric temperatures T1,T2 that produced the largest 3-hour change in density for the period of record 1947-1994.

Fig. 11 ISS orbital decay from three models for altitudes of 400 and $450 \mathrm{~km}$ for beginning date January 22, 1958, a high solar activity case.

Fig. 12 ISS altitude after 100 days of orbital decay from initial altitudes of 400 and 450 $\mathrm{km}$. 


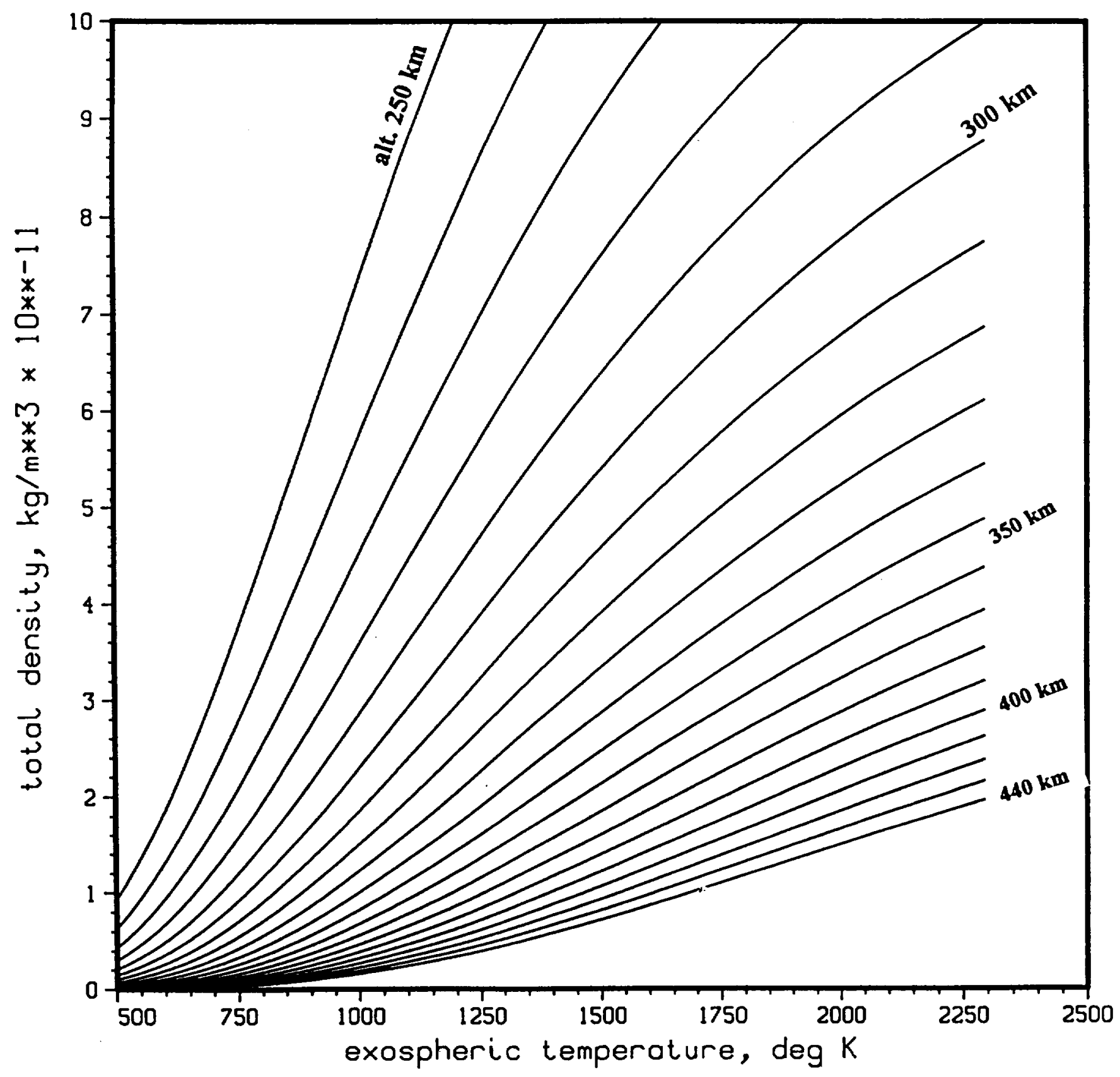




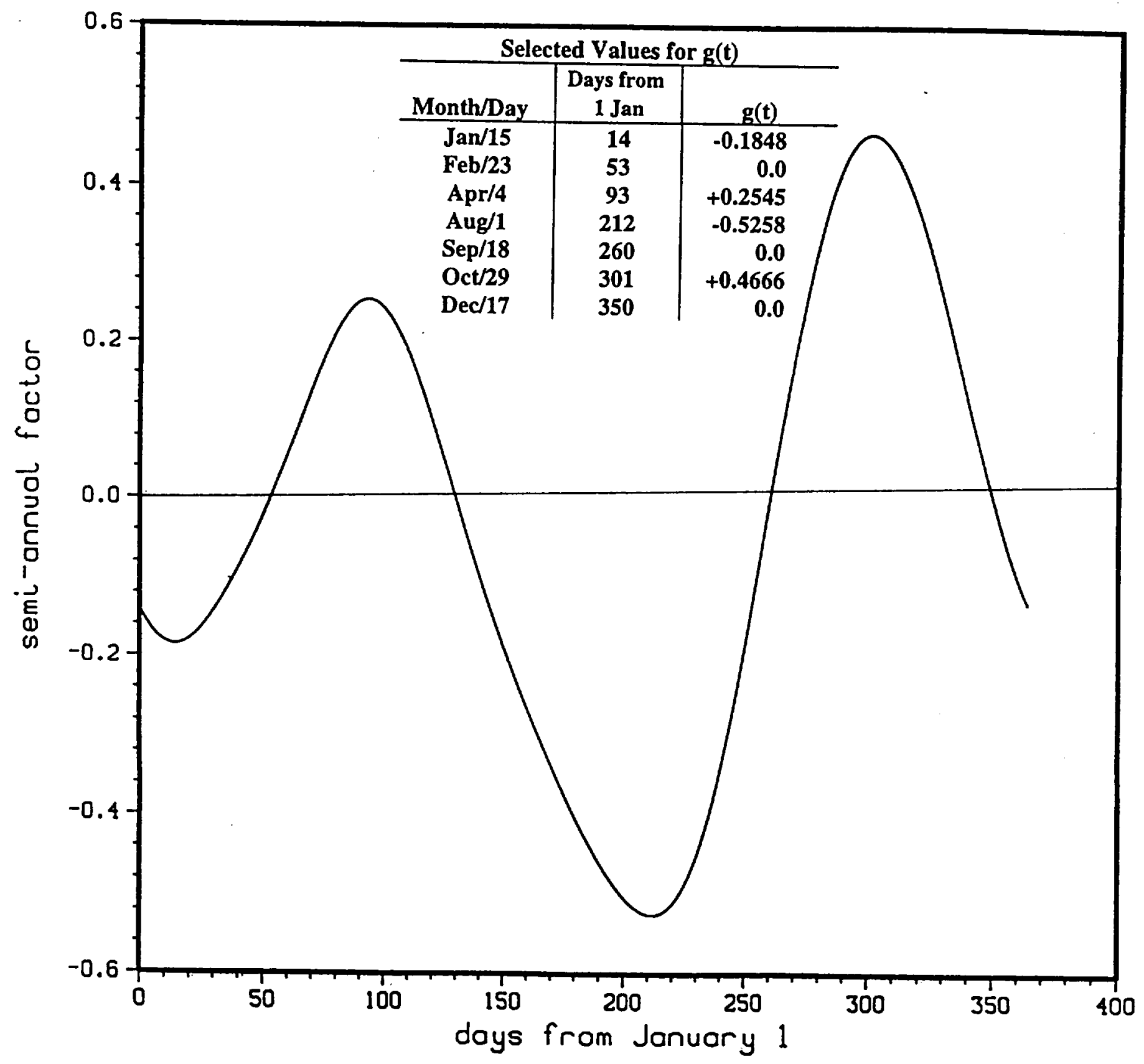




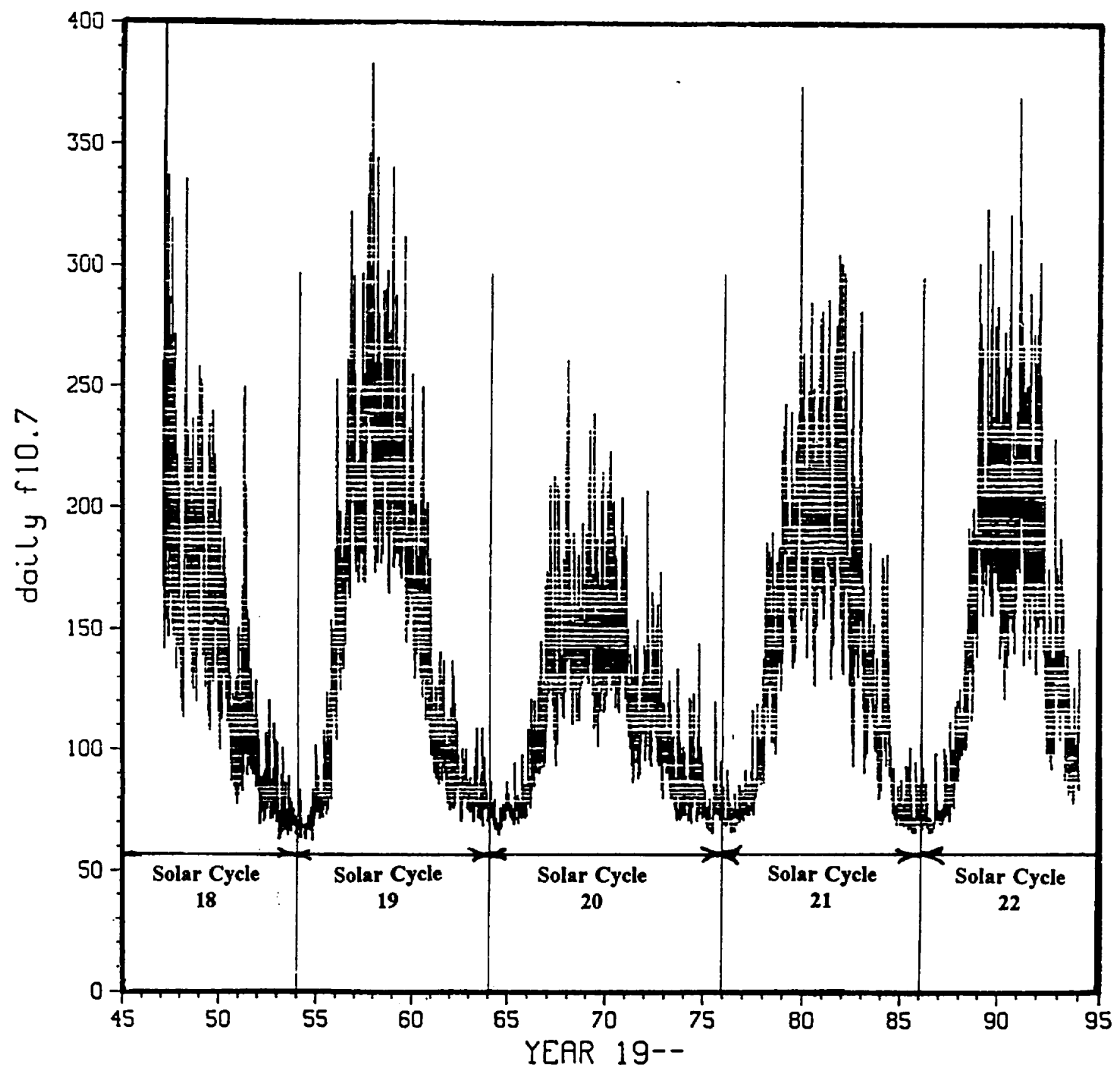









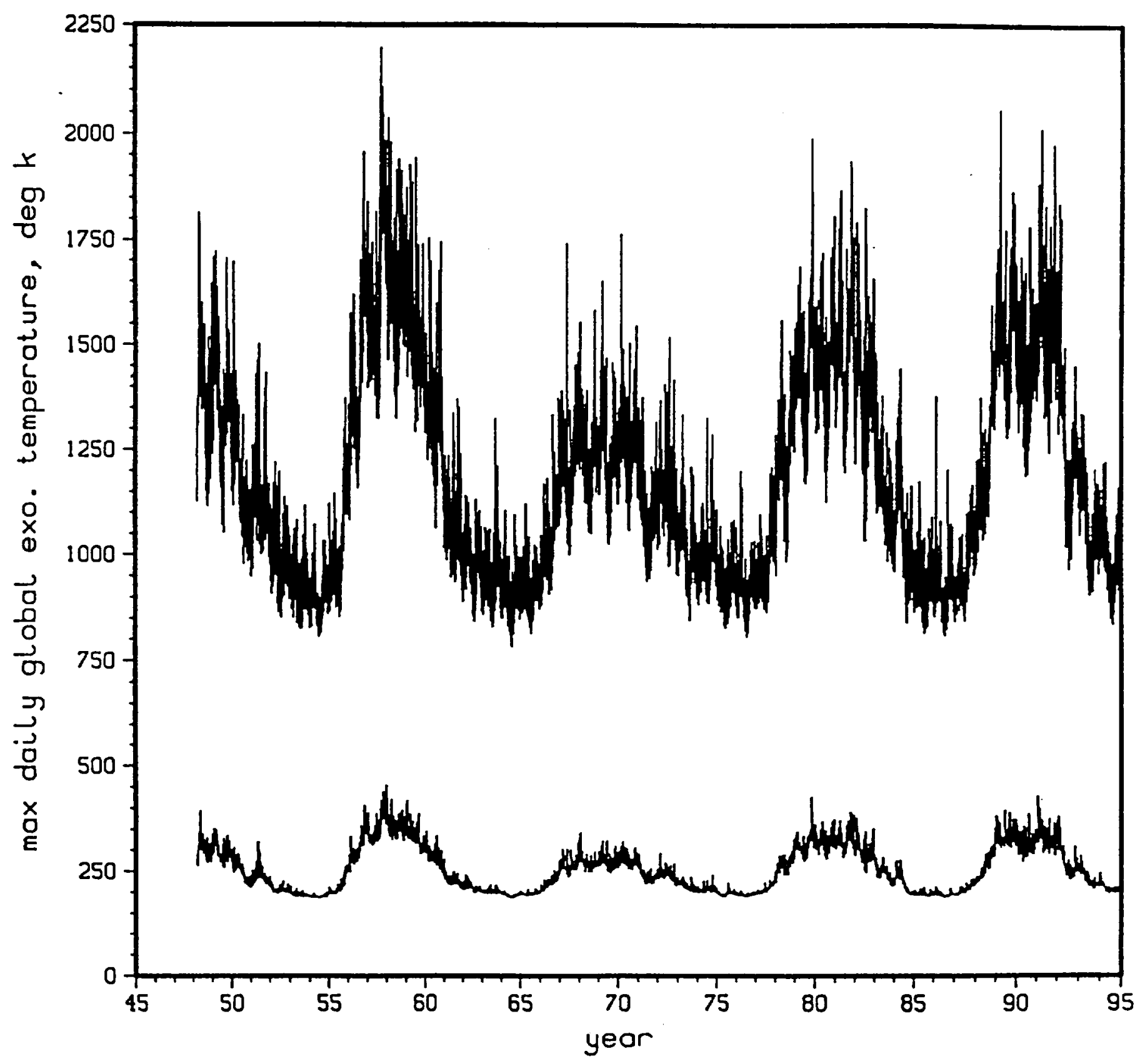




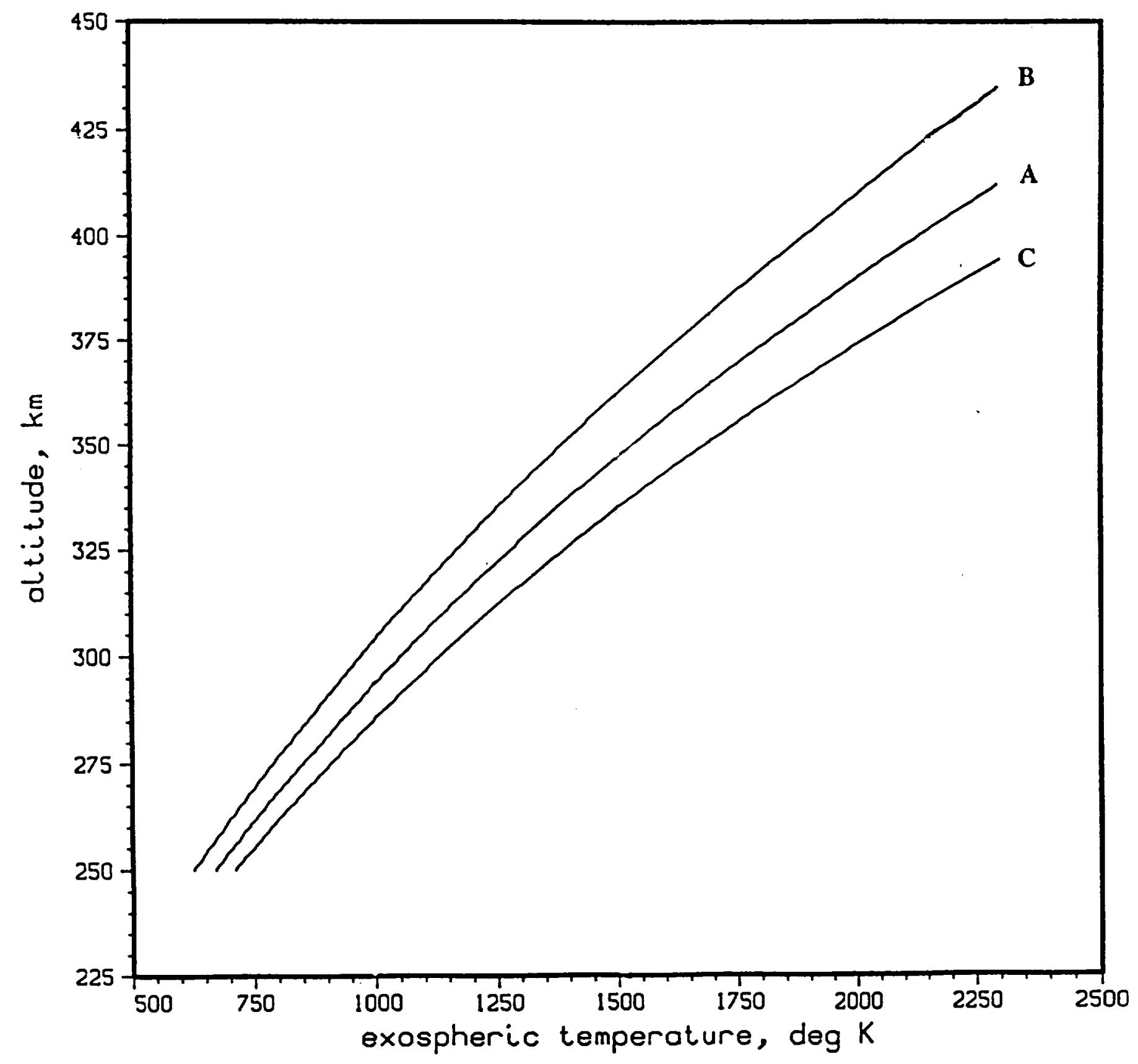




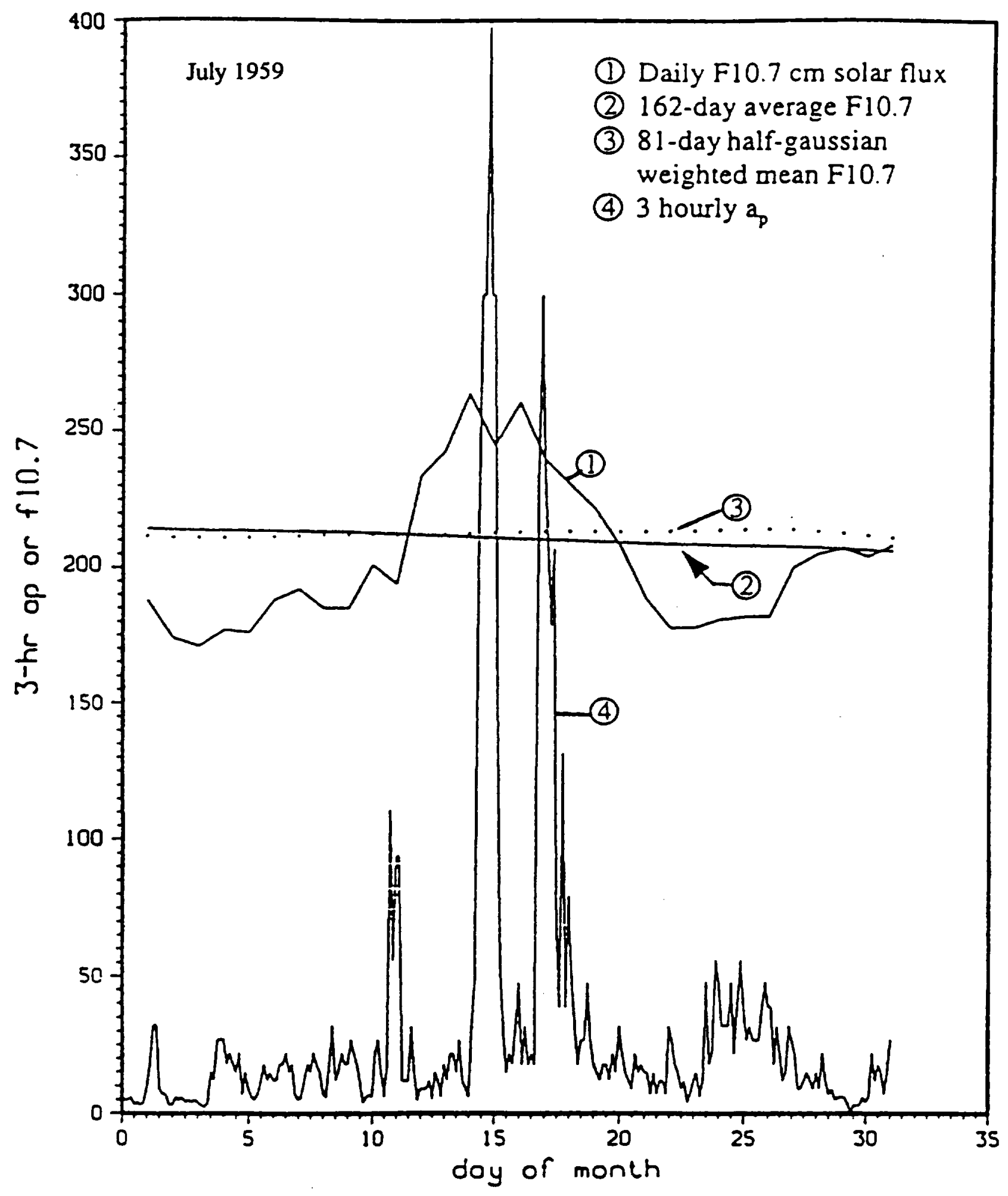




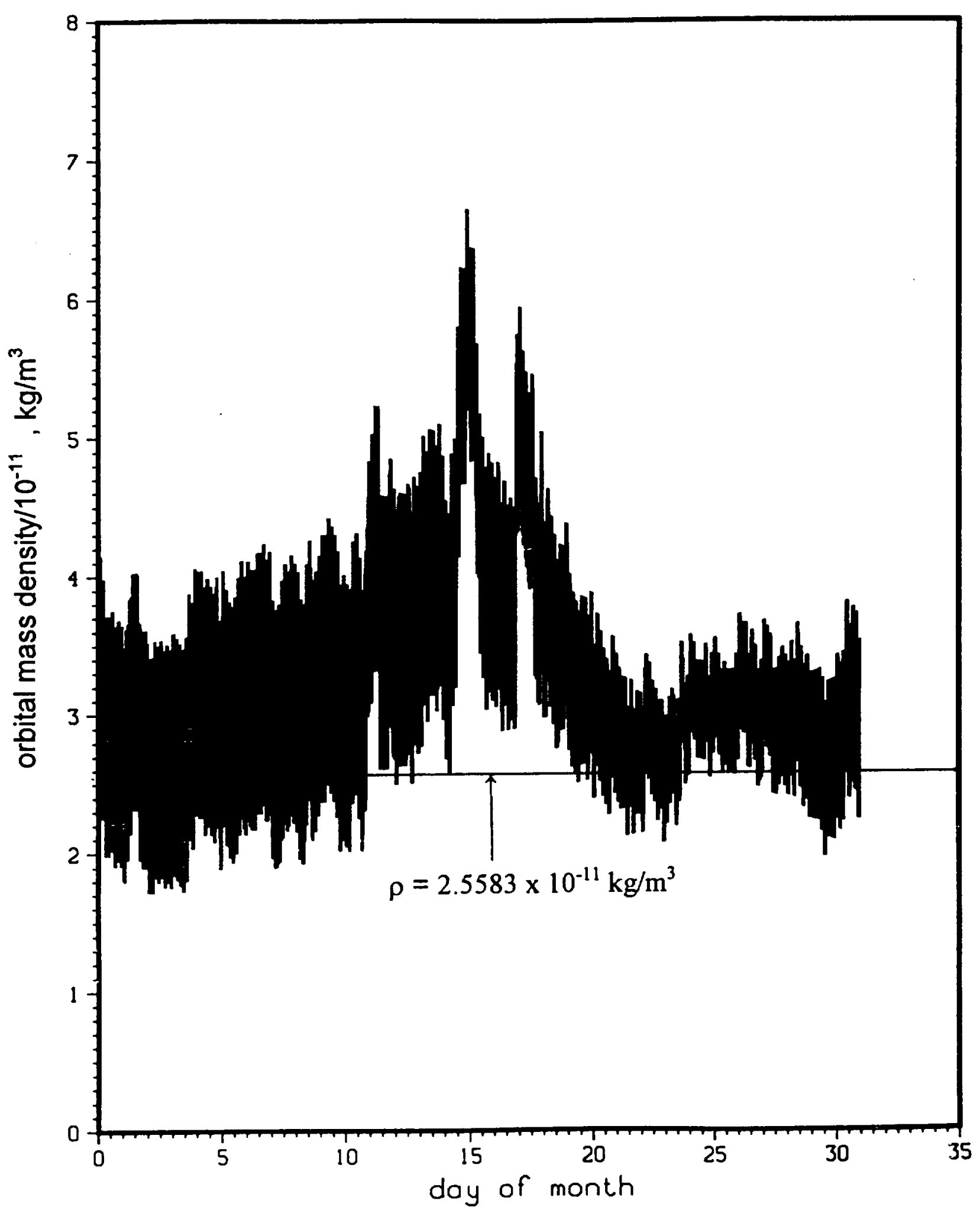




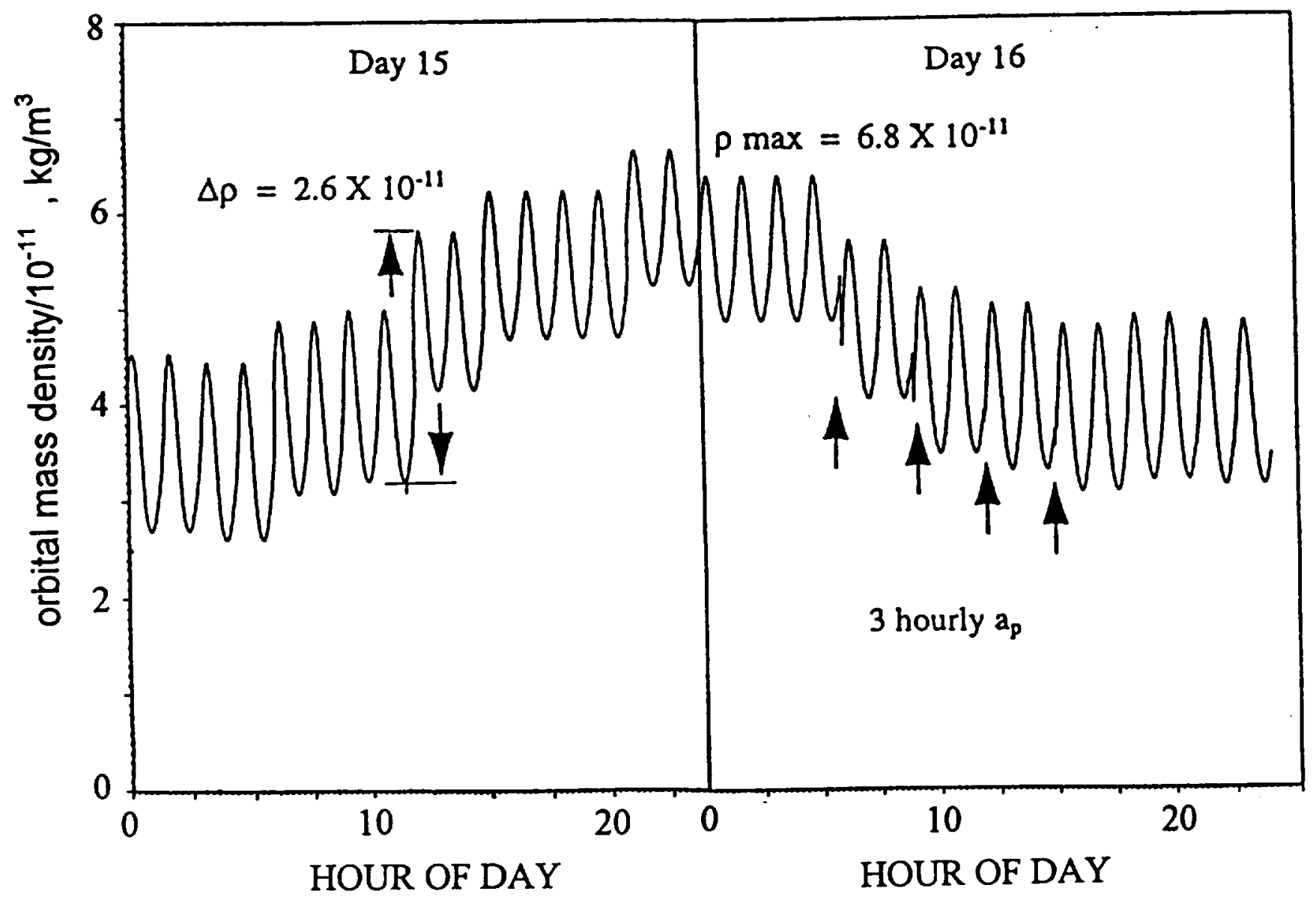




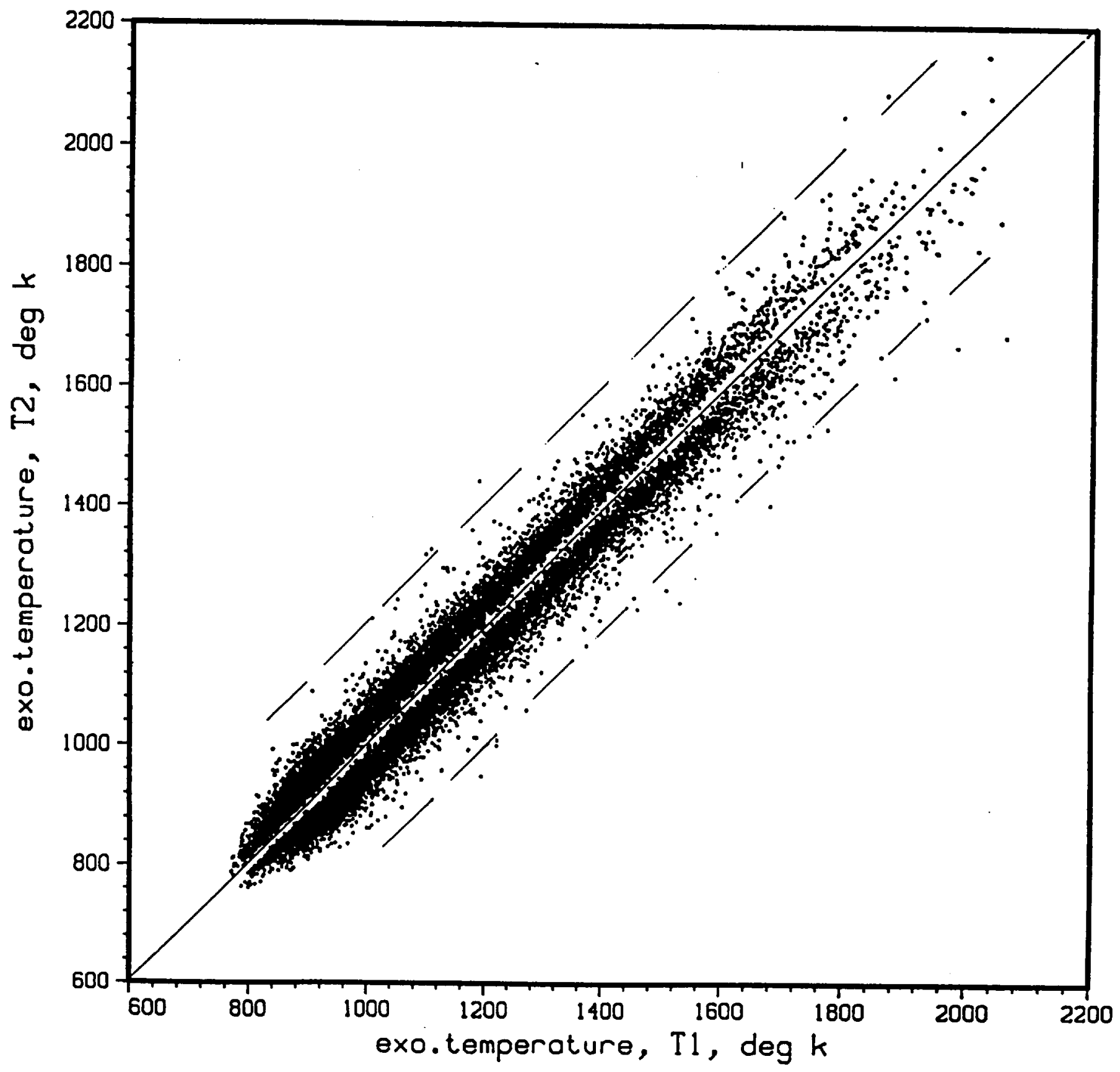




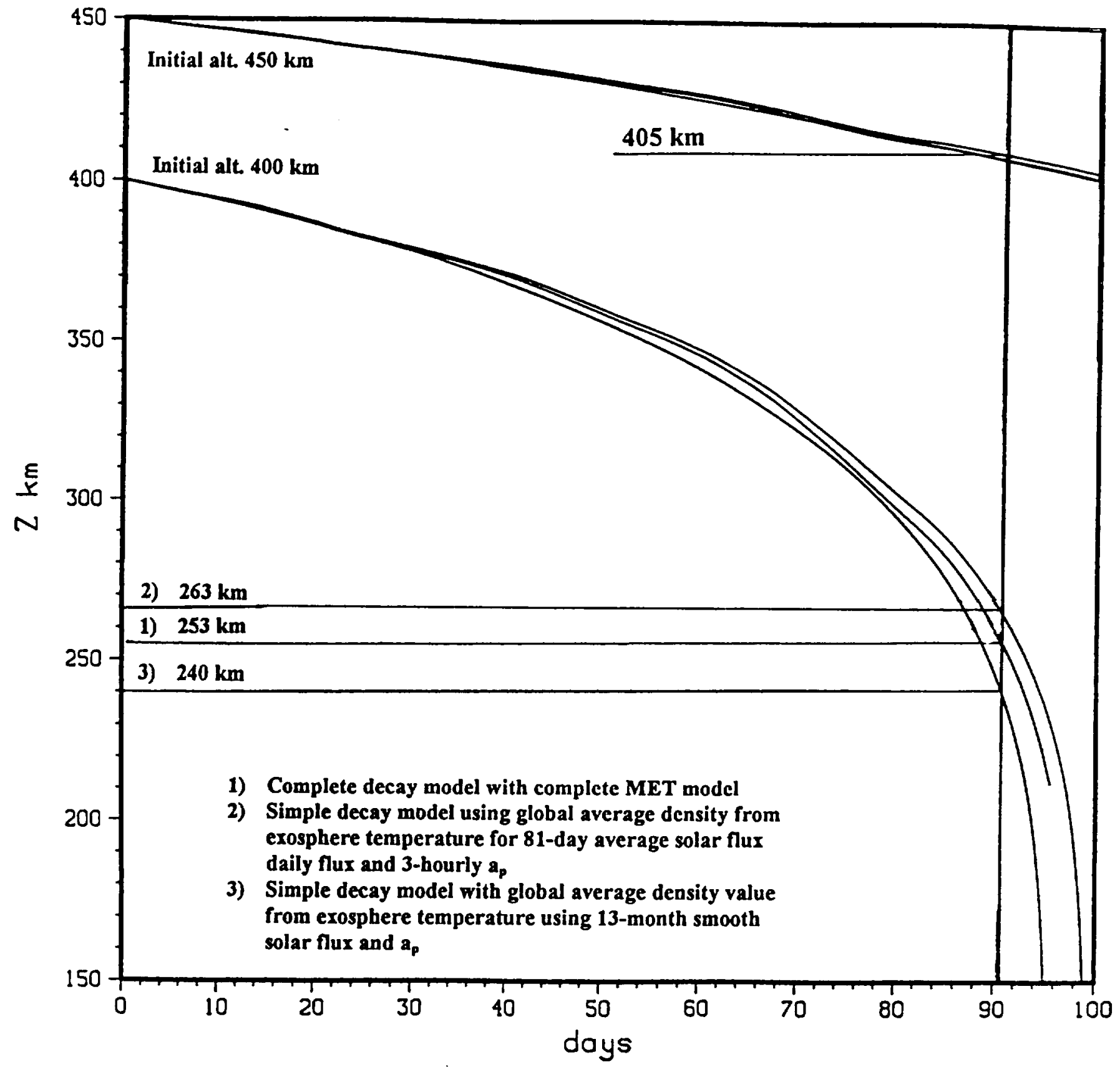






